\title{
A New Fuzzy Ratio and Its Application to the Single Input Rule Modules Connected Fuzzy Inference System
}

\author{
${ }^{1}$ Chian Haur Jong, ${ }^{* 1}$ Kai Meng Tay, ${ }^{2}$ Chee Peng Lim \\ ${ }^{1}$ Faculty of Engineering, Universiti Malaysia Sarawak, \\ Sarawak, Malaysia \\ *kmtay@feng.unimas.my \\ ${ }^{2}$ Centre for Intelligent Systems Research, \\ Deakin University, Australia.
}

\begin{abstract}
The principle of ratios has been applied to many real world problems, e.g. the part-to-part and part-to-whole ratio formulations. As it is difficult for humans to provide an exact ratio in many real situations, we introduce a fuzzy ratio in this paper. We use some notions from fuzzy arithmetic to analyze fuzzy ratios captured from humans. An application of the formulated fuzzy ratio to a Single Input Rule Modules connected Fuzzy Inference System (SIRMs-FIS) is demonstrated. Instead of using a precise weight, fuzzy sets are employed to represent the relative importance of each rule module. The resulting fuzzy weights are explained as a fuzzy ratio on a weight domain. In addition, a new SIRMs-FIS model with fuzzy weights and part-to-whole fuzzy ratio is devised. A simulated example is presented to clarify the proposed SIRM-FIS model.
\end{abstract}

Keywords: Fuzzy ratio, fuzzy arithmetic, single input rule modules connected fuzzy inference system, fuzzy weights

\section{INTRODUCTION}

In general, a ratio is concerned with the relationship of two magnitudes of the same kind, e.g., the size of two objects, or the height of two persons [1]. Two types of ratios are available, i.e., part-to-part and part-to-whole ratios. As an example, there are five students in a class, with two boys and three girls. Based on the principle of the part-to-part ratio, the ratio of boys to girls is $2: 3$. On the other hand, based on the principle of the part-to-whole ratio, the ratio of boys to all students is 2:5 and. Similarly, the ratio of girls to all students is 3:5.

In this paper, we argue that it sometimes is difficult (if not impossible) for humans to provide an exact ratio based on their experience, in some real-world scenarios. A ratio can be imprecise in nature too. As an example, one may suggest that a good micro-nutrient ratio to burn body fat is $30 \%$ protein, $15-20 \%$ fat, $50-55 \%$ carbohydrates [2]. Another example in civil engineering is that to produce an approximately 3000 psi cubic yard of concrete (27 cubic feet), a workable concrete mixture ratio is suggested to be 517 pounds of cement, 1560 pounds of sand, 1600 pounds of stone, and 32-34 gallons of water (or approximately 267.2-283.9 pounds) [3]. In the financial world, an experienced financial advisor would suggest a good debt to income ratio for an individual is near or below $30 \%$ [4].
In this paper, the idea of a fuzzy ratio is introduced. It is argued that one out of two (or both) magnitudes of the same type are known imprecisely, instead of precisely. As such, a fuzzy set is used to represent the magnitude of the same type. Here, the trapezoidal fuzzy set, which is a generalization of the triangular fuzzy set and interval set [5], is used. Some notions from fuzzy arithmetic [5-6] are employed to analyze fuzzy ratios. The validity of the proposed approach is further analyzed mathematically.

A Single Input Rule Modules connected Fuzzy Inference System (here after denoted as SIRMs-FIS) [7-8] is used to demonstrate the applicability of the proposed fuzzy ratio. SIRMs-FIS is a relatively new fuzzy reasoning model in which its final output is obtained by summarizing the product of the importance degree and inference result from single input fuzzy rule modules [7]. In our previous investigations, a new fuzzy failure mode and effect analysis (FMEA) methodology with SIRMs-FIS was proposed [9], and the use of harmony search to optimize an SIRMs-FIS model was demonstrated [10].

One of the issues of SIRMs-FIS is the difficulty to determine the exact (precise) weight for each rule module. The weight can be imprecise in some real-world applications. Therefore, an imprecise weight (i.e., a trapezoidal fuzzy set) is employed to represent the relative importance of each rule module, instead of a precise weight, is suggested. This allows uncertainty of the weights to be included. The weight domain needs to be formally defined. In this paper, these fuzzy weights are explained as a fuzzy ratio pertaining to the weight domain, which can be normalized. A simulated example is presented to clarify the proposed approach. The resulting approach is useful for tackling decision making and assessment problems, e.g., FMEA [9].

This paper is organized as follows. In Section II, trapezoidal fuzzy sets and fuzzy arithmetic are reviewed. In Section III, the idea of fuzzy ratios is introduced, with an example included. In Section IV, the new SIRMs-FIS model with fuzzy weights is explained. Finally, concluding remarks and suggestions for further work are provided in Section V. 